\title{
In-shoe Sensor System with an Embedded User Interface and Wearable Leg Unit
}

\author{
Inês Lourenço, João Costa
}

\begin{abstract}
In-shoe sensor systems are of great interest to monitor foot health, sports activities and rehabilitation strategies. Among the potential users are people with diabetes, a large part of the population for which monitoring foot pressure and temperature is critical to avoid ulceration, and even amputation. Despite all these reasons the use of foot monitoring devices is still uncommon compared to other accessories such as fitness tracking devices. This work describes the development of an instrumented insole for monitoring pressure, temperature and humidity taking advantage of widely available wearable components. This is made possible by additionally developing a shield board for time-division multiplexing of the pressure signals and an embedded user interface which is stored in the microcontroller's memory and uploaded to a smartphone at start-up via Bluetooth Low Energy. The user interface runs on a smartphone to provide both real time monitoring and averages of sensor data. The system is described in detail and validated by monitoring pressure patterns during stance, by testing response to temperature variations and observing patterns in individuals with pes planus posture.
\end{abstract}

\section{INTRODUCTION}

In-shoe sensor systems have long been the focus of research because they have the potential to provide important information related to the foot. Motivations range from early diagnosis of gait pathologies [1], prevention of complications such as ulceration [2], [3], [4], evaluation of the effectiveness of rehabilitation treatments [5] as well as sports training tools to improve athletic performance [6]. Early research can be traced back to 1963, when Bauman and Brand [7] developed a device for plantar pressure measurements to evaluate footwear for patients with leprosy. Since then many other devices were proposed fueled by the arrival of new pressure transducers [8], wireless protocols, miniaturization of electronics and improvements in power efficiency that extend battery life. More recently commercial devices were made available which integrate multiple sensors including pressure sensors and accelerometers [9].

Despite all the advances the adoption of in-shoe monitoring devices remains well below its prognosticated potential. Studies show that up to $25 \%$ of diabetic individuals

Research supported by FCT - Fundação para a Ciência e a Tecnologia, within the Research Unit CTS - Center of Technology and systems, references UID/EEA/00066/2013 and project IPL/2018/I\&D_CTS /UNINOVA_ISEL

Inês Lourenço is a student at ISEL/ESTeSL, Instituto Superior de Engenharia de Lisboa, Instituto Politécnico de Lisboa, R. Conselheiro Emídio Navarro, 1959-007 Lisboa, Portugal.

João Costa is with Instituto Superior de Engenharia de Lisboa, Instituto Politécnico de Lisboa, R. Conselheiro Emídio Navarro, 1959-007 Lisboa, Portugal and also a member of CTS-UNINOVA, Quinta da Torre, Monte da Caparica, 2829-516, Caparica, Portugal (corresponding author e-mail: jcosta@deetc.isel.pt). will develop a foot ulcer in their lifetime and a considerable number ultimately undergo amputation as a result of ulcers that cannot be healed. In principle in-shoe monitoring systems could be implemented to continuously monitor ulceration sites based on known risk factors such as high peak pressure and rising skin temperatures. Globally, an estimated 422 million adults were living with diabetes in 2014 [10] so there is great interest in developing systems that can be widely adopted by the population.

The cost of commercial systems has worked as deterrent, but there is now an opportunity to leverage the development of such devices on increasingly popular wearable electronics components which are almost unobtrusive to the user. Several academic efforts have been made recently to develop solutions with low cost pressure sensing resistors and wireless communication [3], [11], [12]. This work explores such framework by describing the development of an insole based on low cost pressure sensing resistors, an embedded user interface and a Lilypad shield board for time-division multiplexing of the pressure signals. The main difference to previous works is that we focus on a very simple system architecture where the user interface is completely embedded in the microcontroller and the shield board provides the interface between commoditized components.

The device also includes temperature and humidity sensors. Temperature has been shown to be an important parameter, a local temperature increase above $2.2^{\circ} \mathrm{C}$ has been associated to feet lesions [13]. High humidity is also a concern for promoting fungal and bacterial growth which aggravates lesions and delays recovery.

\section{IMPLEMENTATION}

\section{A. The Microcontroller and User Interface}

We have used a simblee ${ }^{\mathrm{TM}}$ module (RFD77101) with a built-in ARM ${ }^{\circledR}$ Cortex M0 microcontroller and Bluetooth Low Energy (BLE) radio transceiver as provided in the Lilypad wearable board format. The module and user interface were completely programmed using the Arduino $\mathbb{R}$ IDE. The user interface code is stored in the microcontroller's program memory and transmitted to the smartphone by BLE at start-up. An application interprets the code and generates the interface in the smartphone display. This process is enabled by the simblee ${ }^{\mathrm{TM}}$ microcontroller on the wearable part and a resident application $\left(\right.$ simblee ${ }^{\mathrm{TM}}$ for mobile) on the smartphone part. The interface was developed using simple draw functions defined in module's software library. The available microcontroller's memory is reduced by the user interface storage but the advantage is that the user-interface is independent of the smartphone operating system and can be easily modified or upgraded without smartphone software reinstallations. With this framework a 
real time in-shoe monitoring interface for mobile phones was created.

The user interface, presented in Fig.1 displays the pressure in six selected points overlaid in an image of the sole of the foot, as well as the humidity and temperature inside and outside the shoe, and the difference between them. The interface is interactive allowing the user to visualize either in real-time mode or on average mode. In the average mode the results presented are averages of the measurements for the complete duration of the monitoring session.

\section{B. Shield Board and Sensors}

The Lilypad format is a popular board format, designed for e-textiles and wearable projects. Lilypad boards can be sewed to clothes and work non-intrusively. However the number of available analog pins is very limited for this type of application. To overcome this problem we have developed a Lilypad shield (a board that can be plugged on top of the Lilypad) for time division multiplexing of the insole pressure signals. This approach allows a single analog pin to serve multiple sensors by sampling them in turn using a multiplexer device.

The complete circuit schematic is presented in Fig.2, the shield board is in the top left division, includes a digitallycontrolled analog multiplexer (MC14053, ON Semiconductor $^{\circledR}$ ), voltage divider resistors for the pressure sensing resistors and pull-up resistors for $\mathrm{I}^{2} \mathrm{C}$ communication with the temperature and humidity sensor device. The board is placed in a leg band, outside the shoe but close to the insole. All necessary resistors are placed in the board to minimize the number of components in the insole.

The shield board is the same size and format as the Lilypad so that it can be aligned on top and connections made by sewing the conductive thread across sew taps on both boards. Referring to Fig. 2, connections to the microcontroller are identified by labels MX, MY and MZ (to 3 analog pins), VDD and GND (to power supply), SDA and SCL (to $\mathrm{I}^{2} \mathrm{C}$ communication pins) and SEL (sensor selection pin). The voltage signals containing plantar pressure information are represented by $\mathrm{S} 1$ to $\mathrm{S} 6$. The digital signal SEL, generated by the microcontroller, toggles state periodically to select which sensors are sampled, either S1, $\mathrm{S} 3, \mathrm{~S} 4$ or S2, S5, S6 in a time division approach.

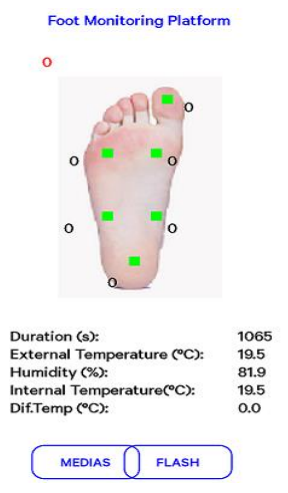

(a)

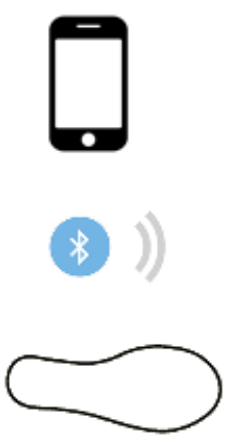

(b)
Figure 1. (a) User Interface as seen in smarphone application. (b) The smartphone receives sensor data using BLE wireless technology.

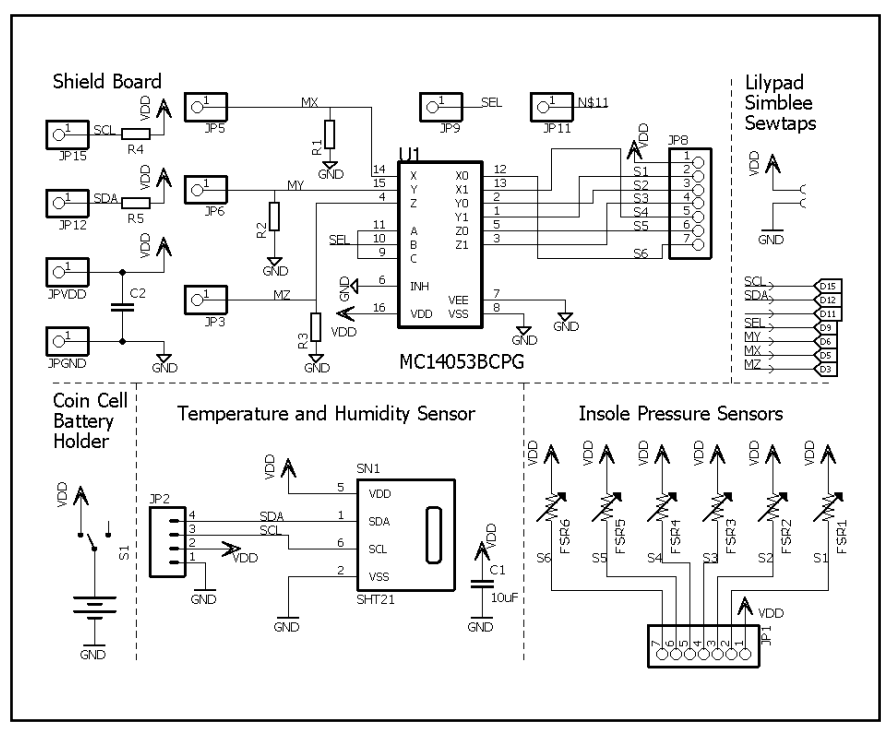

Figure 2. Circuit Schematic with the shielf board (top right), temperature an humidity sensor (bottom center), pressure sensing resistors (bottom right)

In Fig. 2 resistors $\mathrm{R} 1$ to $\mathrm{R} 3$ complete the voltage division circuit for the sensors. The voltage across these resistors is sampled and further processed to determine the resistance of the pressure sensing resistors. To calculate pressure $(P)$ from resistance $(R)$ the microcontroller makes use of a power law curve $P=a R^{b}$ where the parameters $a=73$ and $b=-1.2$ were obtained by fitting the curve to measured data (pressure and resistance given in $\mathrm{kPa}$ and $\mathrm{k} \Omega$, respectively). Optionally plant pressure can be presented in percentage of a maximum reference value. This makes it simple for the user to visualize when the pressure exceeds the reference. The reference is configurable up to $800 \mathrm{kPa}$.

In order to monitor temperature and humidity the SHT21 module (Sensirion) was soldered on an extra-thin $0.2 \mathrm{~mm}$ thick FR4 printed circuit board to facilitate accommodation inside the shoe. This allows the temperature to be recorded in the shoe and compared with the external temperature obtained from the RFD77101 native temperature sensor. Communication with the module SHT21 was done using $\mathrm{I}^{2} \mathrm{C}$ protocol. The microcontroller makes periodic requests to the module for temperature and humidity and updates the user interface.

Double sided printed circuit boards were designed in Eagle Design Software and produced in ISEL's laboratory by the subtractive method. UV light exposure was followed by revelation, chemical etching and component soldering, as usual. For user comfort, connection from the shield board to the edge of the insole was made using flat cables and connection to the sensors inside the insole was done using low resistance stainless steel conductive thread. The conductive thread was sewed to the insole to make connection to pressure sensing resistors of polymer thick film (Interlink Electronics FSR ${ }^{\circledR} 402$ ) with $7 \mathrm{~mm}$ active radius.

\section{System Overview}

The complete system can be seen in Fig. 3 including the user interface running on the smartphone, the leg band and the insole. The leg band contains a battery holder for a $3 \mathrm{~V} \mathrm{Li}$ coin cell, $20 \mathrm{~mm}$ diameter per $3.2 \mathrm{~mm}$ thick, which powers the 
system. The shield board can also be seen in the image, only the Lilypad is occluded in the picture by the shield board itself. We have measured currents up to $5.25 \mathrm{~mA}$ in operation with the BLE connection and the prototype fully working. All components have been sewed with conductive thread to make connections between them and also for the purpose of attachment to the leg band. The insole contains the pressure sensors. The sampling frequency, per sensor, is 75 samples per second at 10 bit resolution, however in the real time mode with updates to the user interface through BLE this number is greatly reduced. In order to extend battery life the pressure points can be averaged and updated only whenever the user connects his smartphone via BLE.

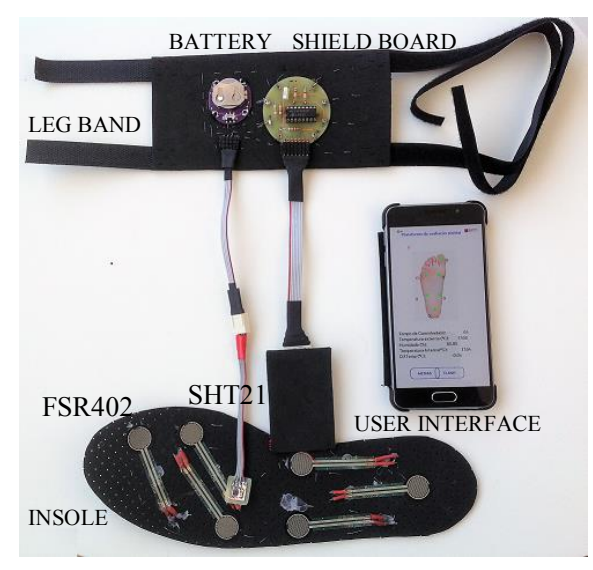

Figure 3. Image of in-shoe sensor system with an embedded user Interface and an wearable leg unit

\section{RESULTS AND DISCUSSION}

The first test was made by visualizing the pressure patterns in the stance phase of the gait cycle. The result is shown in in Fig. 4, left to right, where we first see an image of the heel contact, then an image characteristic of loading to midstance phase, at this stage the pressure is more evenly distributed along the foot, followed by an image of the terminal stance when the heel rises and finally the pre-swing phase when the toes are preparing to rise.

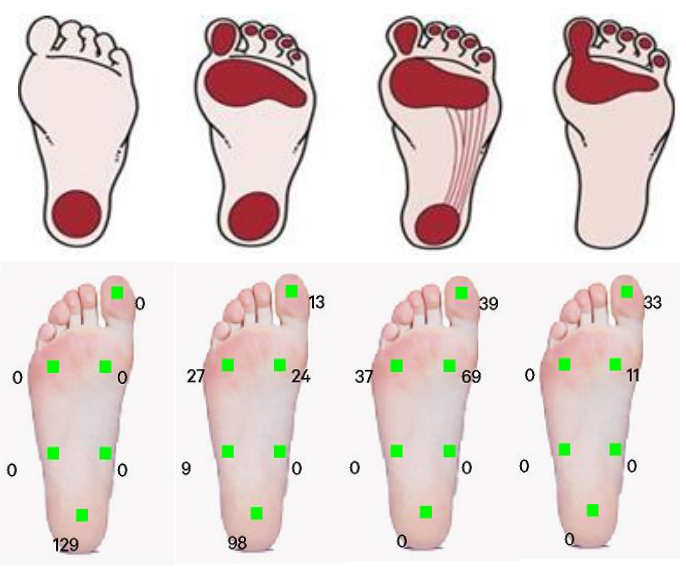

Figure 4. Pressure Tests Stance Phase (kPa)

To test the average monitoring mode, the insole was used for 7 minutes. Average results were recorded and are reported in Table 1 as an example. The results are in line with the expected walking and standing pressure pattern observed in individuals who do not have pes planus (flat feet), displaying low pressure in the inner arch region.

TABLE I. Example of an AVERage Pressure Distribution DURING A MONITORING SESSION

\begin{tabular}{|l|r|}
\hline \multicolumn{1}{|c|}{ Position } & $\begin{array}{c}\text { Pressure } \\
(\boldsymbol{k P a})\end{array}$ \\
\hline Hallux (S1) & 11 \\
\hline $1^{\text {st }}$ Metatarsal (S2) & 19 \\
\hline $5^{\text {th }}$ Metatarsal (S3) & 18 \\
\hline Arch Index (S4) & 5 \\
\hline Inner Arch (S5) & 0 \\
\hline Calcaneus (S6) & 26 \\
\hline
\end{tabular}

An orthostatic test was also done to compare an individual with pes planus versus a control individual with normal feet, the results can be seen in Fig. 5. Although in absolute values the results are different, possibly due to weight and different posture, it can be seen that in pes planus the pressure in the inner side of the foot is relatively higher as would be expected.

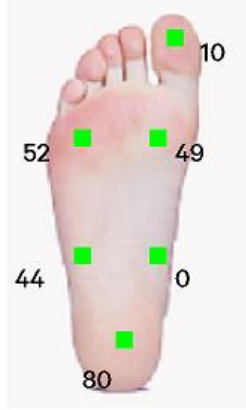

(a)

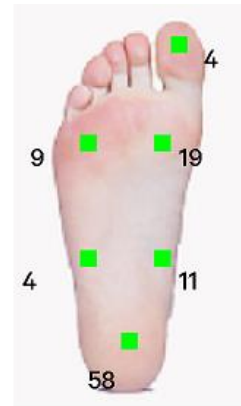

(b)
Figure 5. Orthostatic test. (a) Control and (b) Pes Planus individuals (kPa)

The differential temperature result was tested by thermal heating the region of the sensor and comparing results obtained with a $\mathrm{K}$ thermocouple to find agreement within $4 \%$ for an increase of $15^{\circ} \mathrm{C}$, with room temperature at $21^{\circ} \mathrm{C}$.

As previously reported obtaining a relation between pressure and resistance for polymer thick films is complicated by their non-linear response [3]. The sensors have a threshold behavior in the low pressure range, such that the power law curve fitting, usually applied, does not describe well the behavior below a threshold pressure. We observed such transition at about $2 \mathrm{kPa}$. As a consequence for high resistances (lower pressures) the pressure estimates are below their real values. It is unlikely that this sensor approach could be used to determine quantitatively, with precision, center of pressure's location, ground reaction forces, and other common kinetics parameters. For this 
reason we have focus on a small number of sensors just to provide information about critical pressure points often associated with foot lesions [3]. Nevertheless a qualitative analysis through visualization, as provided in the user display, can provide many insights into abnormal pressure loading and trends over time.

In order to test dynamic response we have recorded pressure in all sensors (S1 to S6) in a time window as shown in Fig.6. The user starts in the sitting positon (stage I), then moves to standing up position (stage II) and next starts walking (stage III). In stage I pressure is low in all sensors except for sensor S6 (heel sensor). When standing the pressure increases for all sensors except for S4 (the inner arch) which always reports low pressure. In the walking stage three pulses can be identified, in each sensor, corresponding to the stance phase of each step. Peak pressure in the heel (sensor S6) precedes peak pressure in the hallux (S1) and metatarsals (S2 and S3) as expected for an individual walking forward. The sensors identification is provided in Table I for reference.
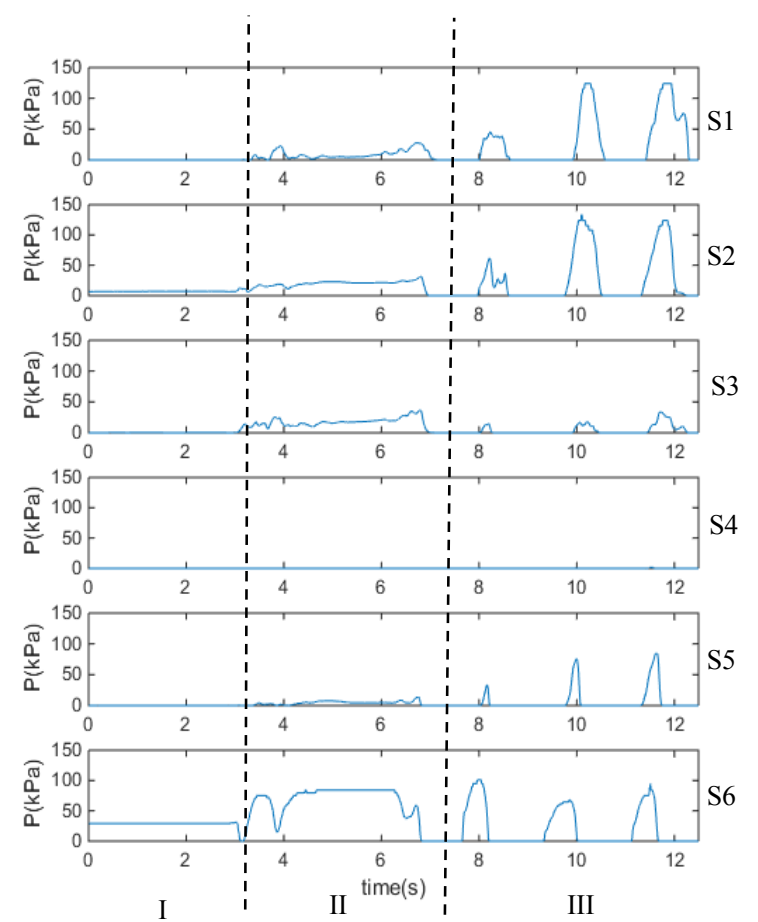

Figure 6. The plots display sensor pressure in a dynamic study during three phases: I - person sitting; II - standing: III- starting to walk (first 3 steps). From top to bottom plots refer to sensors $\mathrm{S} 1$ to $\mathrm{S} 6$ as identified in table I.

\section{CONCLUSION}

The development of an in-shoe prototype for monitoring pressure, temperature and humidity was described. As proofof-concept we have made simple tests to validate the response of the pressure and temperature sensors and the user interface. The goal was to develop a solution using common components available to the wearable electronics community, enhanced by a shield board for time division multiplexing of the pressure signals and an embedded user interface stored in the microcontroller's memory to facilitate monitoring using a smartphone. An important aspect is that offline monitoring is limited, in the future adding an external memory to the microcontroller will enable acquisition during longer periods without BLE. This work is mainly focused on describing the characteristics and architecture of the prototype, further studies will be necessary to validate its effectiveness to identify pes planus and to prevent feet lesions.

\section{REFERENCES}

[1] H. Sobral et al., "Human gait analysis using instrumented shoes," in 2015 IEEE 4th Portuguese Meeting on Bioengineering (ENBENG), 2015.

[2] S. Ostadabbas, A. Saeed, M. Nourani, and M. Pompeo, "Sensor architectural tradeoff for diabetic foot ulcer monitoring," in Proceedings of the Annual International Conference of the IEEE Engineering in Medicine and Biology Society, EMBS, 2012.

[3] A. S. Benbakhti, S. Boukhenous, C. Zizoua, and M. Attari, "An instrumented shoe for ambulatory prevention of diabetic foot ulceration," in Proceedings of the 2014 4th International Conference on Wireless Mobile Communication and Healthcare "Transforming Healthcare Through Innovations in Mobile and Wireless Technologies”, MOBIHEALTH 2014, 2015.

[4] J. H. Low, P. M. Khin, and C. H. Yeow, "A pressureredistributing insole using soft sensors and actuators," in Proceedings - IEEE International Conference on Robotics and Automation, 2015.

[5] L. Pinto, F. Gomes, P. Carvalhais, T. Roseiro, and L. Roseiro, "Pre and post surgery evaluation of patients with hallux pathology using an insoles measurement system of plantar pressure distribution," in ENBENG 2017 - 5th Portuguese Meeting on Bioengineering, Proceedings, 2017.

[6] A. M. Tan, F. K. Fuss, Y. Weizman, and O. Troynikov, "Development of a smart insole for medical and sports purposes," in Procedia Engineering, 2015.

[7] J. H. Bauman and P. W. Brand, "Measurement of Pressure Between Foot and Shoe," Lancet, vol. 281, no. 7282, pp. 629632, 1963.

[8] Z. Geng, M. G. Pepper, and Y. Yan, "Design and characterisation of a single element tri-axial piezoelectric transducer for in-shoe force measurement," in 2010 IEEE International Instrumentation and Measurement Technology Conference, I2MTC 2010 -

Proceedings, 2010.

[9] J. A. Ramirez-Bautista, J. A. Huerta-Ruelas, S. L. ChaparroCárdenas, and A. Hernández-Zavala, "A Review in Detection and Monitoring Gait Disorders Using In-Shoe Plantar Measurement Systems," IEEE Reviews in Biomedical Engineering. 2017.

[10] World Health Organization, Global Report on Diabetes. 2016.

[11] M. Saito et al., "An in-shoe device to measure plantar pressure during daily human activity," Med. Eng. Phys., 2011.

[12] A. M. Tan, F. K. Fuss, Y. Weizman, Y. Woudstra, and O. Troynikov, "Design of Low Cost Smart Insole for Real Time Measurement of Plantar Pressure," Procedia Technol., 2015.

[13] N. Amin and J. Doupis, "Diabetic foot disease: From the evaluation of the 'foot at risk' to the novel diabetic ulcer treatment modalities," World J. Diabetes, 2016. 